\title{
UlRICH HELBACH
}

\section{Der Nachlass Friedrich Schlegels im Historischen Archiv des Erzbistums Köln}

I. Bestandsgeschichte bis zum Erwerb (als Depositum) durch das Historische Archiv

Der anzuzeigende Nachlass ${ }^{1}$ ist Eigentum der Görres-Gesellschaft zur Pflege der Wissenschaft. ${ }^{2}$ Die Görres-Gesellschaft wurde im Jahre I 876 von einer Gruppe katholischer Forscher und Publizisten gegründet. Kurz nach der Gründung erhielt die Gesellschaft durch Vermächtnis einen beachtlichen Teil des Nachlasses von Friedrich Schlegel; ${ }^{3}$ wahrscheinlich durch Vermittlung des Bonner Oberbürgermeisters a. D. Leopold Kaufmann, Mitbegründer und erster Generalsekretär der Görres-Gesellschaft, der mit den Erben Schlegels befreundet war. ${ }^{4}$ Friedrichs Bruder August Wilhelm war seit i 8 I 8 Professor für Literatur in Bonn, wo er I 845 starb.

I Dieser Beitrag ist ein Auszug aus dem neu erstellten Kurzverzeichnis Nachlass Friedrich Schlegel (I772-I 829), bearbeitet von Ulrich Helbach und (zum Biogramm Schlegels sowie zur Liste der Archivalien) Katja Goldmann, Histor. Archiv des Erzb. Köln 2009. Vgl. zum Folgenden die Akte: Histor. Archiv des Erzbistums Köln (AEK), Archiv der Görres-Gesellschaft (GG) I, Akte Nr. I 23 , ferner mündliche und schriftliche Hinweise von Hans Elmar Onnau (übermittelt 2009, vgl. Dienstregistratur des Histor. Archivs des Erzbistums Köln).

2 Vgl. dazu Rudolf Morsey: »Görres-Gesellschaft «. In: Görres-Gesellschaft (Hg.): Staatslexikon - Recht Wirtschaft Gesellschaft. Bd. 2. 7. Aufl. Freiburg i. Br. 1986, S. 1082-1 08 5. Ferner: Rudolf Morsey: »Görres-Gesellschaft «. In: LThK. Bd. 4. 3. Aufl. Freiburg 1995, Sp. 843, bzw.: http://www.goerres-gesellschaft.de/ gesellschaft.html.

3 Vgl. dazu eine Notiz von der Hand der Tochter des Philipp Veit (geb. I9 I2) im Nachlass Veits: 1878 (AEK, GG, Nr. I 23), so auch Alois Dempf: »Vom Schlegelnachlaß der Görresgesellschaft «, in: Historisches Jahrbuch 74 (1955), S. $432-$ 438, nach Josef Körner: Friedrich Schlegel. Neue philosophische Schriften. Frankfurt a. M. 1935, S. 334-336; erwähnt werden mehrere Manuskripte (u. a. »Nachträglicher Zusatz vom ganzen Goethe «, I 824). Das Datum bestätigt auch Hans Elmar Onnau, intimer Kenner der Historie der Görres-Gesellschaft.

4 Freundl. Hinweise hierzu und zum Folgenden von Hans Elmar Onnau (anhand von Forschungen); vgl. auch AEK GG I, Nr. I 23. Kaufmann war mit der Familie Veit befreundet.

Der Nachlass Friedrich Schlegels 
140 Der Nachlass, der I 89 I vom Hause Kaufmanns in das Geschäftszimmer der Görres-Gesellschaft beim Verlag Bachem in Köln verbracht worden war, geriet dort zunächst in Vergessenheit. Wohl aus wissenschaftspolitischen Gründen - so legte es Alois Dempf I 955 im Historischen Jahrbuch dar ${ }^{5}$ - unterblieb damals, unter dem bis I 9 I 9 amtierenden, kulturpolitisch aktiven Präsidenten der Gesellschaft, Freiherr von Hertling, die Auswertung der Manuskripte. Der Teilnachlass, den Dempf »wohl de[n] erheblichste[n] Teil des Nachlasses von Friedrich Schlegel $\ll^{6}$ nannte, entsprach in philosophischer Hinsicht nicht der Linie der traditionellen Philosophie gemäß der damaligen kirchlichen Position des späten I9. Jahrhunderts. So blieb der Nachlass längere Zeit »vergessen im Geschäftszimmer der Gesellschaft liegen $\ll{ }^{7}$

I 93 I kursierte das irrige Gerücht, die Papiere seien verloren gegangen ${ }^{8}$ bzw. bei der Görres-Gesellschaft sei »mit höchster Wahrscheinlichkeit [...] niemals etwas von Schlegel vorhanden gewesen «. ${ }^{9}$ Daher musste 1932 der Schlegelforscher Heinrich Finke in einem Aufsatz im Jahresbericht der Görres-Gesellschaft ausdrücklich nach dem (derzeitigen) Verbleib des an die GörresGesellschaft gelangten Hauptteils des Nachlasses fragen. ${ }^{10}$ I 935 konnte dann Josef Körner konkret auf den Nachlass hinweisen; ${ }^{11}$

5 Vgl. Dempf: »Vom Schlegelnachlaß der Görresgesellschaft « (s. Anm. 3).

6 Ebd., S. 432.

7 Ebd.

8 Vgl. Heinrich Finke 1928 in der Kölnischen Volkszeitung vom 26.9. (Sonntagsbeilage; ein Exemplar in AEK, GG, Nr. I 23 ) über Aussagen (von I 923-1 928 ) von Josef Körner, I 878 habe die Görres-Gesellschaft Manuskripte aus Familienbesitz erhalten, über deren Verbleib die Gesellschaft nichts wisse; vgl. dazu auch AEK GG, Nr. I23, Schreiben von Curtius, I 4.1 0.1931, Spael an H. Finke, 8.10.1931, Körner an N. N. (Geheimrat), I I.2.1932, u. a.

9 Finke 1928 in der Kölnischen Volkszeitung (s. Anm. 8).

I Heinrich Finke: »Der Nachlaß Friedrich Schlegels und die Görres-Gesellschaft. « In: Jahresbericht der Görres-Gesellschaft 1930/3 I ( 1932), S. 80-84.

I I Vgl. Josef Körner: Friedrich Schlegel. Neue philosophische Schriften. Frankfurt a. M. 1935 . 
mit einer genauen Auflistung der Stücke, die der schon zitierte Alois 141 Dempf 1955 erneut aufführte. ${ }^{12}$

Als die I 948 in Köln neu gegründete Görres-Gesellschaft - sie war I 94 I durch das NS-Regime aufgelöst worden - im Jahre I 995 ihr bis 1938 reichendes Archiv (im Umfang von 30 Archivkartons) im Historischen Archiv des Erzbistums Köln deponierte, befand sich darunter nicht der gewichtige Nachlass Schlegel, sondern lediglich ein kleiner Teil desselben. Ausweislich einer Telefonnotiz von $1980^{13}$ wurde damals der Nachlass Schlegel, ebenso wie die Nachlässe Schelling, Eichendorff und Görres, von den jeweiligen wissenschaftlichen Editoren benutzt bzw. lagerten noch bei denselben.

Der Schlegel-Nachlass hatte den Krieg und die NS-Zeit beim Verlag Bachem gut überstanden und wurde erst 1955 durch den Philosophen Alois Dempf - damals Leiter der philosophischen Sektion der Gesellschaft - gewissermaßen wieder entdeckt und durch die Görres-Gesellschaft an ihn ausgeliehen. Dempf reichte die Manuskripte Schlegels für die geplante Gesamtausgabe an seinen Schüler Ernst Behler weiter, einige Teile gingen auch an den Literaturwissenschaftler Hermann Kunisch. Dempf, Behler und Kunisch referierten auf der Generalversammlung der Görres-Gesellschaft I 955 über den Nachlass. ${ }^{14}$ Es folgte die Arbeit an der auf 35 Bände ( 1958 ff.) angelegten Kritischen Friedrich-Schlegel-Ausgabe, die bei Schöningh in Paderborn erscheint. Ausweislich der den Faszikeln beiliegenden Umschläge und Notizzettel waren Unterlagen während der I $960 e r$ Jahre in verschiedenen Teilen durch die Görres-Gesellschaft an den Editor und Hauptherausgeber der Kritischen Friedrich-Schlegel-Ausgabe Ernst Behler bzw. an Ursula Behler $^{15}$ übersandt worden. Die Rückgabevermerke zeigen, dass

I 2 Dempf: »Vom Schlegelnachlaß der Görresgesellschaft « (s. Anm. 3), S. 437 f. Dabei könnten einzelne der genannten Stücke auch erst nach dem Erwerb von I 878/80 hinzuerworben worden sein.

I 3 Dienstregistratur des Historischen Archivs des Erzbistums Köln.

I 4 Vgl. Dempf: »Vom Schlegelnachlaß der Görresgesellschaft « (s. Anm. 3).

I 5 Damals: Bonn-Duisdorf, Maarweg 30.

Der Nachlass Friedrich Schlegels 
142 Behler diese Unterlagen längere Zeit im Besitz hatte. ${ }^{16}$ Behler wechselte 1964 in die USA, an die University of Washington in Seattle, und hinterlegte den Nachlass bei seinem Verleger Schöningh. Als die Schlegel-Bearbeitungen Behlers spätestens mit dem Tode des Forschers 1997 (in den USA) endeten und das Editionsunternehmen ins Stocken geriet, war bei der Görres-Gesellschaft nicht allseits bekannt, wo sich der Nachlass befand.

Über Jahrzehnte hatte der Nachlass dem Editionsprojekt gedient, so dass der Entleiher des Großteils der Papiere, der Verleger Ferdinand Schöningh, sicherlich dankbar war, die Stücke für die Fortführung des in seinem Verlag erscheinenden Unternehmens in greifbarer Nähe zu haben. Gelagert waren die Manuskripte in einem Safe. Das beweist der Umstand, dass sich das Faszikel »Entwurf der historiographischen Betrachtungen [um I 820] « 2008 in einem abgestempelten Post-Versandumschlag von 1976 befand, den Ursula Behler an Gottfried Lehr, Verlag Schöningh, Paderborn, geschickt hatte. Der Umschlag ${ }^{17}$ trägt die Aufschrift »H. Schöningh. Schlegel-M[anu $] s[$ kript $]$. Für den Safe.«

Ein kleiner Teil, nämlich die durch Alois Dempf ausgeliehenen I 5 Manuskripte »Zur Geschichte und Politik « (I 8 I I / I 2- I 828), war allerdings schon Anfang der I $990 e r$ Jahre wieder an die Görres-Gesellschaft gelangt, ${ }^{18}$ die sie ihrem von der Universität München ins Historische Archiv des Erzbistums nach Köln verlagerten Vereins-

I6 Ein Karton trug den Absendervermerk der Wiss. Buchgesellschaft (Darmstadt) und ist an Behler adressiert, doch hat der Karton ursprünglich wohl nicht die Schlegelunterlagen enthalten. Er ist vielmehr von Behler zur Verpackung der Papiere benutzt worden.

17 AEK, Nachlass Schlegel, Nr. 69.

I 8 Man hatte diese Faszikel an die Sektionsleiterin der Historischen Sektion der Görres-Gesellschaft, Laetitia Boehm (München) gegeben. Von dort gelangten die Stücke an Hans Elmar Onnau, den Archivar der Gesellschaft, der sie dem Historischen Archiv des Erzbistums Köln übergab, wo sie dem Archivbestand der Gesellschaft ergänzend beigefügt wurden (als Stücke Nr. 244-258). Die gedruckte Beständeübersicht des Historischen Archivs von 1998 weist diesen Status aus (Das Historische Archiv des Erzbistums Köln. Übersicht über seine Geschichte, Aufgaben und Bestände, erstellt von den Mitarbeiterinnen und Mitarbeitern des Historischen Archivs des Erzbistums KöIn. Redaktion Toni Diederich und Ulrich Helbach. Siegburg 1998, S. 3 I 2 f.) 
archiv eingliederte. Schwierig blieb die Rückgabe des Hauptteils. 143 Erst aufgrund mehrfacher Anfragen von Hans Elmar Onnau u. a. über den damaligen Präsidenten der Gesellschaft, Paul Mikat, konnte schließlich vor einigen Jahren die Zusage des Verlegers erwirkt werden, den Nachlass wieder an den Eigentümer auszuhändigen. ${ }^{19}$

Zur Rückgabe der umfänglichen Unterlagen kam es zunächst aber noch nicht, da der Verleger Ferdinand Schöningh 2004 plötzlich verstarb. Der Witwe war womöglich nicht bewusst, wem und wohin die Schlegeliana gehörten, so dass der Nachlass erst im Frühjahr $2008^{20}$ über einen Umweg, die Akademie der Wissenschaften in Düsseldorf, an die Geschäftsstelle der Görres-Gesellschaft in Bonn, den heutigen Sitz der Organisation, ${ }^{21}$ gelangte. Im Frühjahr 2008 setzte sich der für das Archiv der Gesellschaft zuständige Hans Elmar Onnau beim amtierenden Präsidenten der Gesellschaft, Wolfgang Bergsdorf, erfolgreich für eine Übergabe in das Archiv der Görres-Gesellschaft in Köln ein. ${ }^{22}$ Zwischenzeitlich hatten der Präsident der Friedrich-Schlegel-Gesellschaft und aktuelle Herausgeber der kritischen Schlegelausgabe, Ulrich Breuer (Johannes Gutenberg-Universität Mainz), und seine Mitarbeiterin, Maren Jäger, sich in Bonn nach den Unterlagen erkundigt, um eine Benutzung durch das Editionsprojekt zu erreichen. Am 8. Dezember 2008 überbrachte dann die für die Geschäftsführung zuständige Helena Andres im Auftrag des Präsidenten der Görres-Gesellschaft dem Historischen Archiv diese Stücke in mehreren Pappschachteln (Archiv-Zugang I 567/08). Darunter befand sich auch ein Manuskript (bzgl. Goethe), das schon 2002 von Hermann Kunisch jun. nach Bonn zurückgelangt war.

Für die Überführung des Großteils des Nachlasses Schlegel in eine Archivinstitution war es förderlich, dass 1995 das gesamte Vereins-

19 Seit den I $970 e r$ Jahren hatte schon Rudolf Morsey, seit I 977 Vizepräsident der Görres-Gesellschaft, sich vergebens bemüht, die Rückgabe des gesamten Nachlassteils der Görres-Gesellschaft zu erwirken.

20 Auskunft von Helena Andres (Bonn, Geschäftsführerin der Görres-Gesellschaft).

2 I Ihr Protektor ist derzeit der Kölner Erzbischof Joachim Kardinal Meisner.

22 Korrespondenz in der Dienstregistratur des Histor. Archivs des Erzbistums Köln. 
144 archiv der Görres-Gesellschaft - seit ca. I 997 zusätzlich die von Dempf ausgeliehenen is Schlegelmanuskripte - im Historischen Archiv des Erzbistums Köln als Depositum nicht nur sicher hinterlegt, sondern zugleich professionell betreut und allgemein zugänglich war. ${ }^{23}$

Rund I 30 Jahre nach dem Erwerb des Nachlasses Friedrich Schlegel durch die Görres-Gesellschaft wurde dieser in ein öffentlich nutzbares Archiv übergeben. Der Nachlass hat eine bewegte Geschichte, die es potenziellen Nutzern nicht einfach machte. Bis dato lag er zwar sicher verwahrt, aber ungenutzt - z. T. sogar unbekannt -, dann durch die Editoren intensiv genutzt und gut geschützt, aber für die allgemeine Forschung und Öffentlichkeit nicht greifbar an verschiedenen Orten. Die Archivierung im Historischen Archiv des Erzbistums Köln dient sowohl der sachgerechten Verwahrung dieser wertvollen Kulturschätze als auch ihrer optimalen öffentlichen Nutzung. Sie stehen ab sofort allen Interessenten zur Verfügung.

II. Die Bearbeitung im Historischen Archiv des Erzbistums Köln

Entsprechend der Bedeutung des umfänglichen Teilnachlasses - er umfasst 332 I beschriebene Seiten - wurden die Stücke im Historischen Archiv des Erzbistums Köln zu einem eigenen Archivbestand »Nachlass Friedrich Schlegel (Depositum der GörresGesellschaft) « formiert. Insgesamt i 5 Nachtragsnummern (Nr. 244258) wurden zu diesem Zweck aus dem Findbuch des Bestandes »Archiv der Görres-Gesellschaft « (S. I 22) ausgetragen und den jetzt archivierten Nachlassstücken hinzugefügt. Alle Stücke wurden durch Katja Goldmann (als Praktikantin) und den Autor dieser Zeilen sorgfältig auf beiliegende Metainformationen aus neuerer

23 Das Findbuch der Görres-Gesellschaft, das Martin Schütz (München) und Hans Elmar Onnau (Kerpen) I 992 bzw. 200 I gefertigt haben (Endredaktion 2002), enthält in Form eines Anhangs die I 5 »Manuskripte Friedrich Schlegels im Besitz der Görres-Gesellschaft I 8 I I - I 828 «, wobei auf den Aufsatz von Dempf - »Vom Schlegelnachlaß der Görresgesellschaft « (s. Anm. 3) - verwiesen wird. 
Zeit durchgesehen und dann einzeln aufgelistet (nach einer grob 145 thematischen Gliederung und ohne die für Autographen üblichen Format- und Umfang-Angaben) und in Archivmappen verpackt sowie - erstmals in ihrer Geschichte - mit eindeutigen laufenden Archiv-Nummern versehen. Sämtliche Stücke wurden neu paginiert (durch die Praktikantin Margot Lyautey, Paris), wobei die Zählung i. d. R. auch die Außenumschlagseiten einbezieht. ${ }^{24}$ Sehr wenige Stücke waren durchgehend paginiert. Da, wo alte Paginierungen teilweise vorhanden waren, wurden die neuen Seitenzahlen unterstrichen. Die Stücke wurden mit den Listen von Körner und Dempf abgeglichen. Dabei wurde deutlich, dass von dem ursprünglich vorhandenen Material immerhin noch zehn Positionen ${ }^{25}$ fehlen. Nach ihnen bleibt zu suchen.

\section{Zu den übrigen Teilen des Nachlasses Friedrich Schlegels}

Der Wert des Teilnachlasses der Görres-Gesellschaft liegt in den Manuskripten und Skizzen aus Schlegels Schaffen. Zwar sind die Schriften Schlegels vielfach gedruckt. Doch enthalten die überlieferten Manuskripte und Skizzen vielfach Entwürfe bzw. Texte mit ergänzenden Bearbeitungen.

Hefte zu verschiedenen Schaffensbereichen sind in beachtlicher Menge vorhanden, in deutscher, aber auch in französischer Sprache. Es dürfte sich - betrachtet man ausschließlich die Entwürfe bzw. Manuskripte Schlegels selbst - um einen wesentlichen Teil der Schlegelschriften (Autographen) handeln.

24 Schließlich übertrug Christian Kau (Praktikant) die Anzahl der Seiten auch in die Find-Liste. Die Archivierungsarbeiten waren damit zeitnah (Ende Februar 2009) abgeschlossen.

25 »Zur Geschichte, Okt. I 809.« - »Desgl., I 8 I o.« - »Zur österreichischen Geschichte I, I 807 (historische Gedanken, politische Gedanken.) « - »Zur Historie, I 807, zur Romanze.« - »Zur Historie. « - Z Zu Karl dem Vten 2.« - »Karl der Vte.« - »Gedanken auf der Reise während des Krieges I 809. « - »Versuche, das wesentliche der christlichen europäischen Staatsanstalten im allgemeinen zu entwickeln.« - 》Die Verfassungsmäßigkeit (Erzählung).« - In einem weiteren, elften Fall ließ sich die Titelangabe nicht klar identifizieren: » Indisches, englisch und deutsch.

\section{Der Nachlass Friedrich Schlegels}


146 Folgende andere Institutionen verwahren Nachlass-Teile (abgesehen von Briefen ${ }^{26}$ ) von Schlegels Hand: ${ }^{27}$

Frankfurt, Freies Deutsches Hochstift Frankfurt am Main (Goethe-Haus):

ein Karton ( 79 Nummern $^{28}$ ) mit ( I.) wissenschaftlicher Prosa (Philosophie des Lebens, philos. Vorlesungen, Poesie des Schönen, The protestant apology, Indologisches, betr. Methodisten, Aphoristische Gedanken) und (2.) Korrespondenz (20-30 Briefe) Schlegels (z. B. Schlegel an Novalis), $1793 \mathrm{ff.}^{29}$ (Dauerleihgabe, augenscheinlich auch aus anderer Provenienz [nicht aus dem Nachlass]).

\section{Stadtbibliothek Trier:}

I $7 \mathrm{Hefte}^{30}$ (4-48 Bll.) zur Poesie, Literatur, Philologie und zur griechischen Poesie, I794-I 8 I 2 (Signatur Hs. 2506) (erworben I 896 durch Friedrich van Hoffs).

26 Die Korrespondenzen Schlegels finden sich, wie üblich, in anderen Nachlässen.

27 Die Auflistung kann und möchte nicht sämtliche Nachlassteile nachweisen, sondern dient der Einordnung des hier vorliegenden Teilnachlasses. Wie verstreut der Nachlass ist, erweist der Blick in die zentrale Nachlass-Datenbank des Bundesarchivs http://www.nachlassdatenbank.de. Dort wird (im Januar 2009) eine wissenschaftliche Einrichtung ausgewiesen, die einen Karton mit wissenschaftlicher Prosa und Korrespondenz besitzt, nämlich das Freie Deutsche Hochstift, Frankfurt a. M. Daneben befänden sich weitere (kleinere) Nachlassteile in der Staatsbibliothek zu Berlin Preußischer Kulturbesitz und in der Universitätsbibliothek Dresden. Nicht genannt werden mindestens drei weitere Einrichtungen mit Schlegel-Nachlassautographen, darunter die Görres-Gesellschaft. Vgl. zu den verschiedenen Teilnachlässen auch http://www.kalliope-portal.de, wo der Nachlass der Görres-Gesellschaft schon längere Zeit aufgeführt war (ohne nähere Hinweise). Zur frühen Nutzung der anderen Nachlassteile vgl. z. B. in den Schriften der Görres-Gesellschaft: Heinrich Finke: Über Friedrich und Dorothea Schlegel. Köln I91 8.

28 Diese Angabe nach http://www.kalliope-portal.de (Januar 2009).

29 Auskunft Hans Grüters, Frankfurt a. M. (Januar 2009).

$30 \mathrm{Vgl}$. aktuell das maschinenschriftliche Verzeichnis der nicht in den gedruckten Handschriftenkatalogen beschriebenen Handschriften der Stadtbibliothek Trier. Ferner Ernst Behler: »Der Wendepunkt Friedrich Schlegels. Ein Bericht über unveröffentlichte Schriften Friedrich Schlegels in Köln [im Stadtarchiv] und Trier «. In: Philosophisches Jahrbuch der Görres-Gesellschaft 64 ( 1956 ), S. 245271, hier: S. 260 f. 
Berlin, Staatsbibliothek Preußischer Kulturbesitz:

7 Hefte $^{31}$ (3 I-48 Bll.) mit Aufzeichnungen und Notizen zur Literatur und Poesie, I 783 -I 823.

Dresden, Staats- und Universitätsbibliothek:

Ein Manuskript über heilmagnetische Behandlung, ein Verzeichnis ausgeliehener Bücher, ferner Korrespondenzen der Korrespondenzpartner Schlegels (v. a. August Wilhelm Schlegel).32

\section{Universitätsbibliothek Leipzig:}

Zwei Manuskripte: »Von den Schulen der griechischen Poesie « und $\gg$ Vom aesthetischen Werth der Griechischen Komödie «.

IV. Verzeichnis der Archivalien im Einzelnen (Anhang) ${ }^{33}$

i. Unterlagen zur Philosophie

I Manuskript: 1 . Vorlesung [= Philosophie der Sprache (Schlegels letzte Vorlesung), Heft I] (32 Seiten, davon 30 beschrieben)

2 Manuskript: 2. Vorlesung [= Philosophie der Sprache (Schlegels letzte Vorlesung), Heft 2] (32 Seiten, davon 30 beschrieben)

3 Manuskript: 3. Vorlesung [= Philosophie der Sprache (Schlegels letzte Vorlesung), Heft 3 ] (36 Seiten, davon 32 beschrieben)

4 Manuskript: 4. Vorlesung [= Philosophie der Sprache (Schlegels letzte Vorlesung), Heft 4] ( 36 Seiten, davon 33 beschrieben)

5 Manuskript: 5. Vorlesung [= Philosophie der Sprache (Schlegels letzte Vorlesung), Heft 5] (36 Seiten, davon 33 beschrieben)

6 Manuskript: 6. Vorlesung [= Philosophie der Sprache und des Wortes (Schlegels letzte Vorlesung), Heft 6] (36 Seiten, davon 33 beschrieben)

3 I Vgl. www.manuscripta-mediaevalia.de/hs/katalogseiten/HSKo539_boo I_jpg. htm (Januar 2009).

32 Auskunft aus dem Autographenzettelkatalog (Thomas Haffner, Dresden) (Januar 2009).

33 An der Auflistung wirkte maßgeblich Katja Goldmann (s. Anm. I) mit.

Der Nachlass Friedrich Schlegels 
1487 Manuskript: 7. Vorlesung [= Philosophie der Sprache und des Wortes (Schlegels letzte Vorlesung), Heft 7] (36 Seiten, davon 29 beschrieben)

8 Manuskript: 8. Vorlesung [= Philosophie der Sprache und des Wortes (Schlegels letzte Vorlesung), Heft 8] (32 Seiten, davon 30 beschrieben)

9 Manuskript: 9. Vorlesung [= Philosophie der Sprache und des Wortes (Schlegels letzte Vorlesung), Heft 9] (32 Seiten, davon 29 beschrieben)

ıo Manuskript: Io. Vorlesung [= Philosophie der Sprache und des Wortes (Schlegels letzte Vorlesung), Heft ro] (32 Seiten, davon 7 beschrieben) [Bricht nach wenigen Seiten, auf S. 7, mitten im Satz mit Schlegels letzten Worten ab.]

I I Manuskript: Philosophische Fragmente, Erste Epoche I(86 Seiten, davon 17 beschrieben)

I 2 Manuskript: Philosophische Fragmente, Erste Epoche II (96 Seiten, davon 93 beschrieben)

I 3 Manuskript: Philosophische Fragmente, Erste Epoche III (80 Seiten, davon 78 beschrieben)

I 4 Manuskript: Philosophische Fragmente, Zweite Epoche I 96 Seiten, davon 94 beschrieben)

I 5 Manuskript: Philosophische Fragmente, Zweite Epoche II (9o Seiten, davon 88 beschrieben)

I6 Manuskript: Zur Philosophie, nro. I. Paris I 802 Jul. (46 Seiten, davon 45 beschrieben)

I7 Manuskript: Zur Philosophie, nro. II. Paris I 802 Dec. (48 Seiten, davon 45 beschrieben)

I 8 Manuskript: [Philosophische Fragmente, I796, Beilage V] (38 Seiten, davon 37 beschrieben)

I9 Manuskript: Zur Philosophie, nro. III. Paris I 804 Jan. (48 Seiten, davon 47 beschrieben) 
20 Manuskript: Zur Philosophie, I 806 I (auf dem Titelblatt: Schreib- 149 Übungen zur arabischen und indischen Schrift) (64 Seiten, davon 62 beschrieben)

2 I Manuskript: Zur Philosophie, I805 I (48 Seiten, davon 47 beschrieben)

22 Manuskript: Zur Philosophie, I 805 II 72 Seiten, davon 70 beschrieben)

23 Manuskript: Zur Philosophie, I 806 II (78 Seiten, davon 77 beschrieben)

24 Manuskript: Gedanken [ I 808/o9, Beilage IX der Philos. Lj.] (60 Seiten, davon 58 beschrieben)

25 Aufzeichnungen/Notizen in Französisch v. a. zur Philosophie [?] (44 Seiten, davon 16 beschrieben)

26 Manuskript: Philosophie des Lebens (40 Seiten, davon 9 beschrieben)

27 Manuskript: Themata über die Vorlesungen über die Philosophie des Lebens ( 46 Seiten, davon 16 beschrieben)

28 Prospektur [Erläuterungen] über den Inhalt der Vorlesungen über die Philosophie des Lebens (2 Seiten, beschrieben)

29 Manuskript: nicht näher bestimmt (in Französisch), z. B. Kapitel Metaphysique, Morale ( 80 Seiten, davon 54 beschrieben)

\section{ii. Unterlagen zur Literatur}

30 Manuskript: Grundriss der Literatur (48 Seiten, davon 24 beschrieben)

3 I Manuskript: Prinzipien der Literatur (48 Seiten, davon 3 I beschrieben)

32 Manuskript: Geschichte der Literatur. Neue Ausgabe I 820 (32 Seiten, davon 26 beschrieben) 
15033 Manuskript: Darstellung der alten und neuen Literatur. Zusätze zur neuen Ausgabe I 82 I (62 Seiten, davon 57 beschrieben)

34 Manuskript: Nachträglicher Zusatz vom ganzen Goethe in der jetzigen deutschen Literatur I 823 ( 36 Seiten, davon 26 beschrieben)

35 Exzerpte aus Gedichten von Opitz (28 Seiten, davon 27 beschrieben)

36 Manuskript/Notizen über die biblische und hebräische Literatur (30 Seiten, davon 24 beschrieben)

iii. Unterlagen zur Grammatik (Sprache)

37 Manuskript: Breviarium Ambrosianum, Deutsche Grammatik I, Germanische Altertümer, Köln I 804 I (60 Seiten, davon 56 beschrieben)

38 Manuskript: Deutsche Grammatik I, I 805 Jun. Geschichte (z. B. Isländisch, Angelsächsisch, » Köllnisch « ( 56 Seiten, davon 42 beschrieben)

iv. Unterlagen zur Geschichte und Politik

39 Manuskript: Entwurf der Histor. Betrachtungen [um i 820 ] (62 Seiten, davon 60 beschrieben)

40 Manuskript: Fragmente zur Geschichte der Griechischen Poësie (80 Seiten, davon 78 beschrieben)

4I Manuskript: Studien des Alterthums ( 102 Seiten, davon 75 beschrieben)

42 Manuskript: Zur Geschichte und Politik I $8_{I I}$ I $_{1} 8_{\text {I }}$ bis Dec. (Sign. ca. 1997-2008: Archiv der GG 244) (64 Seiten, davon 62 beschrieben)

43 Manuskript: Zur Geschichte und Politik ${ }_{1}$ I $_{3}$ (Dec. I 8 I 2 ) (Sign. ca. 1997-2008: Archiv der GG 245) (94 Seiten, davon 93 beschrieben) 
44 Manuskript: Zur Geschichte und Politik I 8 I 3 II (Sign. ca. I997-2008: 151 Archiv der GG 246) (der Bezug der beiden letzten - losen - Blätter zu dem Stück ist unklar) (98 Seiten, davon 92 beschrieben)

45 Manuskript: Zur Geschichte und Politik I 8 I 5 I (Sign. ca. I 9972008: Archiv der GG 247) (44 Seiten, davon 33 beschrieben)

46 Manuskript: Zur Geschichte und Politik 18 I 6 I (Sign. ca. 19972008: Archiv der GG 248) ( 56 Seiten, davon 54 beschrieben)

47 Manuskript: Zur Geschichte und Politik 1816 II (Sign. ca. 19972008: Archiv der GG 249) (40 Seiten, davon 38 beschrieben)

48 Manuskript: Zur Geschichte und Politik ${ }_{1} 8$ I 7 I (Sign. ca. I 9972008: Archiv der GG 250) (92 Seiten, davon 90 beschrieben)

49 Manuskript: Zur Geschichte und Politik 18 I 8 I (Sign. ca. 19972008: Archiv der GG 25 I) ( 72 Seiten, davon 70 beschrieben)

50 Manuskript: Zur Geschichte und Politik I 82 I (Sign. ca. I9972008: Archiv der GG 252) (62 Seiten, davon 60 beschrieben)

5 I Manuskript: Zur Geschichte und Politik I 823 (Sign. ca. 19972008: Archiv der GG 253) (60 Seiten, davon 58 beschrieben)

52 Manuskript: Zur Geschichte und Politik 1824 (Sign. ca. I 9972008: Archiv der GG 254) (anscheinend fehlt am Ende I Seite) (62 Seiten, davon 6I beschrieben)

53 Manuskript: Zur Geschichte und Politik I 826 [I] (Sign. ca. I 9972008: Archiv der GG 255) (8 I Seiten, davon 79 beschrieben)

54 Manuskript: Zur Geschichte und Politik I 826 II (Sign. ca. I 9972008: Archiv der GG 256) (76 Seiten, davon 74 beschrieben)

55 Manuskript: Zur Geschichte und Politik 1827 I (Sign. ca. 19972008: Archiv der GG 257) (96 Seiten, davon 94 beschrieben)

56 Manuskript: Zur Geschichte und Politik r 828 I Juli (Sign. ca. 1997-2008: Archiv der GG 258) (80 Seiten, davon 74 beschrieben) 
152 v. Unterlagen zu Ägypten, Indien und Orient (Sprache, Kultur etc.)

57 Manuskript: Précis du système hiéroglyphique des anciens Egyptiens ... (über die altägyptische Schrift) [ 1 824?] (I 20 Seiten, alle beschrieben)

58 Manuskript: Indische Untersuchungen (Entwurf), I 823 (es fehlt offenbar I Blatt am Ende) (62 Seiten, davon 60 beschrieben)

59 Manuskript: History of Sacontola from the Mahabharat by Wilkins. Oriental Repertory [o. D.] (38 Seiten, davon 35 beschrieben)

60 Manuskript: Orientalische Gedanken (u. a. über arabische Schrift und Sprache), Nov. I 805 (42 Seiten, davon 39 beschrieben)

61 Manuskript: Orientalia I 806 (mit einigen Handzeichnungen/Skizzen) ( 72 Seiten, davon 70 beschrieben)

62 Manuskript: Anmerkungen und Auszüge zu den Studien des Altertums ( 64 Seiten, davon 5 beschrieben)

63 Manuskripte: Über die Sprache und Weisheit der Indier (I) (70 Seiten, davon 68 beschrieben)

64 Über die Sprache und Weisheit der Inder II (es fehlt offenbar I Blatt am Ende) (62 Seiten, davon 60 beschrieben)

65 Manuskript/Abschrift (Schlegels): Narodos Rede (40 Seiten, davon 37 beschrieben)

66 Abschrift (Schlegels) des Rechtskodex: The laws of Menu, Son of Brahma. Chapter the first. On the .... With a summary of contents [o. D.] ( 14 Seiten, alle beschrieben)

vi. Unterlagen zu Sonstigem (bzw. nicht näher identifizierte)

67 Kleinformatiges Manuskript (Notizen): Sylvester (22 Seiten, davon I I beschrieben)

68 Faszikel (4 Seiten, zusammenhanglos) aus einem Manuskript (Thematik: Religion/Philosophie) (4 Seiten, alle beschrieben)

(das Stück lag lose in Nr. 34 (betr. Goethe), evtl. von einem der Nutzer nachträglich dort eingelegt) 
69 Metainformationen zur Bearbeitung / Lagerung / Archivierung der Nachlass-Unterlagen Schlegels seit den I $950 e r$ Jahren: Notizen, Umschläge und ähnliches

70 Metainformationen zur Bearbeitung / Lagerung / Archivierung der Nachlass-Unterlagen Schlegels seit den I $950 e r$ Jahren: Kartonagen, in denen der Nachlass Ende 2008 dem Histor. Archiv des Erzbistums überbracht wurde. 